\title{
Futuristic Nanomaterials and Composites: Part II
}

\author{
CHANG-SOO KIM, ${ }^{1}$ DIRK LEHMHUS, ${ }^{2,3}$ JAMES NJUGUNA, ${ }^{4}$ \\ MURALIDHARAN PARAMSOTHY, ${ }^{5,6,8}$ and MEISHA LEI SHOFNER ${ }^{7}$
}

\begin{abstract}
1.-Materials Science and Engineering Department, University of Wisconsin-Milwaukee, $3200 \mathrm{~N}$ Cramer Street, Milwaukee, WI 53211, USA. 2.-ISIS Sensorial Materials Scientific Centre, University of Bremen, Wiener Strasse 12, 28359 Bremen, Germany. 3.-MAPEX Center for Materials and Processes, University of Bremen, Bibliothekstraße 1, 28359 Bremen, Germany. 4.-Centre for Advanced Engineering Materials, School of Engineering, Robert Gordon University, Garthdee House, Garthdee Road, Aberdeen AB10 7QB, UK. 5.-School of Science \& Technology, Singapore Institute of Management University (UNISIM), 461 Clementi Road, Singapore 599491, Singapore. 6.-NanoWorld Innovations (NWI), 1 Jalan Mawar, Singapore 368931, Singapore. 7.-School of Materials Science and Engineering and Renewable Bioproducts Institute, Georgia Institute of Technology, 801 Ferst Drive, Atlanta, GA 30332-0295, USA. 8.—e-mail: mpsothy@yahoo.co.uk
\end{abstract}

\section{INTRODUCTION}

The present special topic "Futuristic Nanomaterials and Composites: Part II" is a continuation of last month's topic "Futuristic Nanomaterials and Composites: Part I". In this continuation, inspiration is once again drawn from two conferences: (1) NANOSTRUC 2014: International Conference on Structural Nano Composites held in Madrid (Spain) on May 20-21, 2014, and (2) Nanocomposites III symposium of the 2015 TMS Annual Meeting \& Exhibition held in Orlando, Florida (USA), on March 15-19, 2015. By linking these two events, this joint special issue manifests a trans-Atlantic networking in the field of nanocomposites which will be strengthened further in upcoming events of both series. The following paragraphs describe the 16 articles in this continuation based on a division between structural/tribological on the one hand and functional aspects on the other, with one contribution combining both.

\section{STRUCTURAL/TRIBOLOGICAL ASPECTS}

Akpan et al.'s work, titled "Mechanical Behavior of Electrospun Palmfruit Bunch Reinforced Polylactide Composite Fibers", examines the mechanical properties of electrospun palm fruit bunch reinforced poly lactic acid (PLA) nanofiber composites using treated and untreated fillers. PLA reinforced with treated palm fruit bunch samples had better

Chang-Soo Kim, Dirk Lehmhus, James Njuguna, Muralidharan Paramsothy, and Meisha Lei Shofner are the guest editors for the Composite Materials Committee, a joint committee of the TMS Functional Materials Division (FMD) and the TMS Structural Materials Division (SMD); and coordinators of the topic Futuristic Nanomaterials and Composites: Part II in this issue. moisture resistance than PLA reinforced with untreated palm fruit bunch. The moisture resistance of the electrospun fibers was improved by chemical treatment of the reinforcement. The higher the weight fraction of filler (treated and untreated) in the PLA matrix, the higher was the formation of beads resulting in diminishing tensile strengths. The optimal mechanical responses were obtained at $3 \mathrm{wt} . \%$-treated palm fruit bunch content in PLA. Electrospun nonwoven mats have found applications in areas like filters, sensor devices, electrical conductors, protective cloths, wound dressings, tissue engineering, vascular grafts, and drug delivery systems, among others.

In Jamaati et al.'s work, "Effect of Particles on Continuous and Discontinuous Recrystallization of Nanostructured Inclusion Free Steel", the effect of SiC micro- and nano-particles on continuous and discontinuous dynamic recrystallization of nanostructured interstitial free (IF) steel is investigated. The IF steel discontinuously recrystallized to form new grains during accumulative roll bonding (ARB) at room temperature. Nuclei formed in the area around the $\mathrm{SiC}$ particles and at grain boundaries during ARB. The presence of $\mathrm{SiC}$ particles in the IF steel matrix retarded recovery and led to retention of stored energy from ARB, providing the driving force for discontinuous dynamic recrystallization (DDRX).

Shunmugasamy et al.'s work, "Cellular Magnesium Matrix Foam Composites for Mechanical Damping Applications", is a timely presentation and review of the damping properties of lightweight metal alloys and their composites. Cellular metallic materials (foams) are observed to possess similar or higher damping properties than the corresponding 
dense matrices. This implies that closed-cell foams granted more control over mechanical and damping properties than open-cell and solid particulate composites. A good damping characteristic of metal alloys and metal-matrix composites is viewed as beneficial to the automotive, aerospace, and marine sectors. Use of lightweight materials in this field supports increasing payload capacity and decreasing fuel consumption.

Yousef et al.'s work, titled "Wear Characterizations of POM reinforced with Carbon Nanotubes (POM/CNT) Using Paraffin Oil Dispersion Technique", investigates the influence of carbon nanotubes (CNT) on the specific wear rate of POM/CNT nanocomposites. The POM nanocomposites which were synthesized contained $0.001,0.01,0.02$, and 0.03 wt.\% CNT. The specific wear rate of POM having 0.03 wt.\% CNT in air and water medium was improved by $73 \%$ and $66 \%$, respectively, compared to virgin POM. Here, strength and modulus rose by about $31 \%$ and $29 \%$, respectively, while crystallinity and melting temperature both increased. The higher percentage of CNT provided a better reduction in specific wear rate, and the air medium provided the greatest reduction in wear rate, while the oil medium had the least influence. Adding CNTs to POM increases its proliferation in wear applications involving various friction levels.

In Mehedi et al.'s work, "Improved Wear Resistance of Al-Mg Alloy with $\mathrm{SiC}$ and $\mathrm{Al}_{2} \mathrm{O}_{3}$ Particle Reinforcement", the effects of $\mathrm{SiC}$ and $\mathrm{Al}_{2} \mathrm{O}_{3}$ reinforcement on the tribological property of Al-Mg alloy were investigated. It was found that $\mathrm{SiC}$ particles promoted wear resistance, and that the predominant wear mechanism was abrasion and delamination. Increased load altered the wear mechanism from abrasion to sub-surface crack and delamination.

\section{FUNCTIONAL ASPECTS}

In Granados et al.'s work, "Synthesis of $\mathrm{Zn}_{1-x} \mathrm{Co}_{x} \mathrm{Al}_{2} \mathrm{O}_{4}$ Spinel Nanoparticles by Liquid-Feed Flame Spray Pyrolysis: Ceramic Pigments Application", $\mathrm{Zn}_{1-x} \mathrm{Co}_{x} \mathrm{Al}_{2} \mathrm{O}_{4}$ nanopowders were produced by a liquid-feed flame spray pyrolysis (LF-FPS) method, using metal-organic precursors as starting materials. The resulting spinel nanopowders were evaluated in terms of phase, color, size, and morphology. The results suggest that LF-FPS is an excellent alternative method to produce ceramic pigments of nanometer size and spherical shape, with the added possibility to obtain new hues and bright colors, to which considerable market potential for industrial applications is attributed.

Jiang et al.'s work, titled "Electronic Structure of Copper Decorated Carbon Nanotubes", studies the electronic and transport properties of $(5,5)$ armchair carbon nanotubes (CNTs) encapsulated by 1 , 2,4 , and $5 \mathrm{Cu}$ chains. Density functional theory (DFT) coupled with non-equilibrium Green's functions (NEGF) was used. The copper-CNT interaction did not form a bond between the two partners. However, their interaction did lower the gap in the CNT's density of states (DOS) around the Fermi level, as well as enhancing the DOS around this same level. The copper-encapsulated CNT $(5,5)$ hybrid system had a greater conductance than the pristine CNT $(5,5)$. The conductance could be improved by $250 \%$ with 5 copper chains in the system.

Simien et al.'s work, "The Role of Percolation Theory in Developing Next Generation Smart Nanomaterials", evaluates the electrical noise characteristics of thin film random networks of single-walled carbon nanotubes with lengths of $820 \mathrm{~nm}, 210 \mathrm{~nm}$ and $130 \mathrm{~nm}$, in addition to mixedlength and pure semiconducting single-walled carbon nanotube networks. This study represents one of the first experimental studies in which highly characterized length-sorted single-walled nanotube networks have been investigated to isolate their contributions to $1 / \mathrm{f}$ noise. Percolated networks of SWNTs with shorter lengths are also shown to be more successful in detecting cracks in concrete structures. This may enable the replacement of traditional strain sensors for monitoring system states. The effect of nanotube length and the associated properties of the composite have to be further investigated, but hold promise for the development of embedded sensors.

In Pan's work, titled "Facile Preparation of $\mathrm{Ag} /$ $\mathrm{NiO}$ Composite Nanosheets and Their Antibacterial Activity", nanoscale, sheet-like precursors of $\mathrm{NiO}$ and $\mathrm{Ag} / \mathrm{NiO}$ with different $\mathrm{Ag}$ contents were synthesized via an easily controlled hydrothermal method. The antibacterial activity of $\mathrm{NiO}$ and $\mathrm{Ag} /$ $\mathrm{NiO}$ composites to several Gram-positive and Gramnegative bacteria was examined. $\mathrm{NiO}$ nanosheets showed hardly any antibacterial activity, but $\mathrm{Ag} /$ $\mathrm{NiO}$ composite nanosheets displayed increased activity even at low $\mathrm{Ag}$ content levels.

Bogovic et al.'s work, "The Controlled Single-Step Synthesis of $\mathrm{Ag} / \mathrm{TiO}_{2}$ and $\mathrm{Au} / \mathrm{TiO}_{2}$ by Ultrasonic Spray Pyrolysis (USP)", investigates the synthesis of $\mathrm{Ag} / \mathrm{TiO}_{2}$ and $\mathrm{Au} / \mathrm{TiO}_{2}$ nanoparticles using the single-step USP process in which their morphology (size and distribution of $\mathrm{Ag} / \mathrm{Au}$ nanoparticles on the oxide surface) was controlled by the right choice of process parameters. In the case of $\mathrm{Ag} / \mathrm{TiO}_{2}$ nanoparticles, almost all the $\mathrm{Ag}$ nanoparticles were formed on the oxide surface $(>90 \%)$, whereas in the case of $\mathrm{Au} / \mathrm{TiO}_{2}$ nanoparticles, some of the $\mathrm{Au}$ nanoparticles were formed within the $\mathrm{TiO}_{2}$ nanoparticles $(\sim 30 \%)$ with random distribution throughout the oxide matrix. Catalysis, sensors, biomaterials, electronics are some of the potential areas of application of these nanocomposite materials.

Mukherjee et al.'s work, "Tuning the Magnetic Properties of Cobalt-Based Metallic Glass Nanocomposites", investigates the temperature- 
induced variation in the magnetic properties of cobalt-based metallic glass. It was demonstrated that a drastic change in magnetic properties for a Co-based metallic glass could be achieved by controlled devitrification involving nanocrystallite formation. However, remnant magnetization decreased on further heating due to grain growth. Tunable magnetic properties with controlled devitrification could lead to potential futuristic applications including magnetic switches and sensors.

In Pillai et al.'s work, titled "Influence of $\mathrm{Pr}$ Doping on the Structural, Morphological, Optical, Luminescent, and Nonlinear Optical Properties of RF Sputtered ZnO Films", the nanoscale and microscale effects of Pr doping on the structural, morphological, optical, and non-linear optical properties of $\mathrm{ZnO}$ film are investigated. Pr doping of hexagonal wurtzite $\mathrm{ZnO}$ crystalline phase enhanced the transparency of the film. A green emission was observed in all the films where intensity increased with Pr concentration. The non-linear absorption behavior switched from saturable absorption (SA) to reverse saturable absorption (RSA) as the Pr doping concentration increased. Pr-added $\mathrm{ZnO}$ nanostructured ceramics is considered to be a potential material for varistor applications including optoelectronic device applications.

In Djekoun et al.'s work, "Synthesis and Characterization of High-Energy Ball-Milled Nanostructured $\mathrm{Fe}_{25} \mathrm{Se}_{75}, \mathrm{Fe}_{25} \mathrm{Se}_{75}$ nanostructured alloy powders were synthesized from elemental $\mathrm{Fe}$ and Se powders using high-energy ball milling, and the morphology of the milled powders were investigated. After $6 \mathrm{~h}$ of milling, crystalline Se was transformed to the amorphous state. The interdiffusion of $\mathrm{Fe}$ and Se led to the formation of different phases: $\mathrm{FeSe}_{2}, \beta$-FeSe, and $\mathrm{Fe}_{7} \mathrm{Se}_{8}$ structures. At $52 \mathrm{~h}$ of milling, $\mathrm{FeSe}_{2}$ was still present, but as the minority phase. Fe-Se alloys have attracted considerable interest based on optical, magnetic, and electrical properties in view of solar cell, magnetic, magneto-electronic, low-temperature superconductor, and optoelectronic device applications.

Elnaz Esmizadeh et al.'s work, "Thermal and Morphological Study of Epoxy Matrix with Chemical and Physical Hybrid of Nanoclay/Carbon Nanotubes", compares physical and chemical hybrids of carbon nanotubes (CNTs) and nanoclay (NC) in epoxy. The advantages and uniqueness in thermal properties was most pronounced in the chemical rather than physical hybrid of nanoclay/carbon nanotubes in epoxy. Synergistic effects of CNT and NC were higher in the chemical hybrid compared to the physical one. The improvement was related to the $3 \mathrm{D}$ structure of the chemical hybrid. In recent years, much attention has been given to the preparation of high-performance polymer nanocomposites containing a combination of nanofillers with different dimensions towards automotive and aerospace transport applications.
Chandan et al.'s work, "Synthesis of Graphene from Used Battery Electrode", studied the economical synthesis of graphene via an electrochemical exfoliation technique using six different aqueous electrolytes. It was observed that anodic exfoliation occurred in all the cases because of hydroxylation and carboxylation reactions through water hydrolysis. The addition of $\mathrm{NaCl}$ to the electrolyte significantly enhanced the yield of the exfoliated product. Graphene has received wide technological attention owing to its exceptional optical, mechanical, thermal, and electronic properties, which have applications in fields such as fuel cells, solar cells, lithium ion batteries, gas sensors, super-capacitors, composite materials, photo-catalysis, and drug delivery applications.

\section{STRUCTURAL AND FUNCTIONAL COMBINED ASPECTS}

Sayyar et al.'s work titled "Synthesis and Characterization of Covalently Linked Graphene/Chitosan Composites" investigated chitosan covalently linked to chemically converted graphene (CCG) and the associated properties. A significant improvement in the thermal stability, tensile strength, and modulus of the composite was observed with increasing graphene content. Also, six orders of magnitude improvement was observed for the conductivity of the polymer when $3 \mathrm{wt} . \%$ graphene was added. The covalently linked graphene/chitosan composites with enhanced mechanical and electrical characteristics are excellent candidates for use in structures for biomedical applications.

Works by Granados et al., Jiang et al., and Simien et al. were presented at the 2015 TMS Annual Meeting in the framework of the symposium Nanocomposites III, while the studies by Esmizadeh et al. and Sayyar et al. formed part of the NANOSTRUC 2014 Conference. All other articles were openly solicited through this journal's annual call for special topics synchronized with the TMS Annual Meeting.

\section{SUMMARY AND OUTLOOK}

Nanoscale particles and structures possess the ability to modify crystallographic structure and associated mechanical properties. Nanoscale particles and structures also possess the ability to modify electronic structures and associated functional (electrical, magnetic, optical) properties. This has relevant applications in modern and futuristic technologies with reference to microelectronics, smart technology, biomedical/antibacterial, magnetic, tribological and structural applications, especially if both crystallographic and electronic structures are simultaneously modified by nanoscale additions. 
The NANOSTRUC 2016 Conference, the third event in this series, is planned for September 12-14, 2016 at Robert Gordon University, Aberdeen, Scotland. The Nanocomposites IV symposium will follow at the TMS 2017 Annual Meeting \& Exhibition, to be held February 26-March 2, 2017, in San Diego, California, USA.

The following papers being published under the topic "Futuristic Nanomaterials and Composites: Part II" provide excellent details and research on the subject. To download any of the papers, follow the url http://link.springer.com/journal/11837/68/1/ page/ 1 to the table of contents page for the January 2016 issue (vol. 68, no. 1).

- "Mechanical Behavior of Electrospul Palmfruit Bunch Reinforced Polylactide Composite Fibers" S.O. Adeosun, E.I. Akpan, O.P. Gbenebor, A. A. Peter, and Samuel Adebayo Olaleye

- "Effect of Particles on Continuous and Discontinuous Recrystallization of Nanostructured IF Stee" Roohollah Jamaati, Mohammad Reza Toroghinejad, Sajjad Amirkhanlou, and Hossein Edris

- "Cellular Magnesium Matrix Foam Composites for Mechanical Damping Applications" Vasanth Chakravarthy Shunmugasamy, Bilal Mansoor, and Nikhil Gupta

- "Wear Characterizations of POM Reinforced with Carbon Nanotubes (POM/CNT) Using Paraffin Oil Dispersion Technique" Samy Yousef, A.M. Visco, G. Galtieri, and James Njuguna

- "Improved Wear Resistance of Al-Mg Alloy with $\mathrm{SiC}$ and $\mathrm{Al}_{2} \mathrm{O}_{3}$ Particle Reinforcement" Md. A. Mehedi, K.M.H. Bhadhon, and M.N. Haque

- "Synthesis of $\mathrm{Zn}_{1-x} \mathrm{Co}_{x} \mathrm{Al}_{2} \mathrm{O}_{4}$ Spinel Nanoparticles by Liquid-Feed Flame Spray Pyrolysis: Ceramic Pigments Application" Natalia Betancur Granados, Eongyu Yi, Richard M. Laine, and Oscar Jaime Restrepo Baena
- "Electronic Structure of Copper-Decorated Carbon Nanotubes" Jingyin Jiang, Chengyu Yang, and Quanfang Chen

- "The Role of Percolation Theory in Developing Next-Generation Smart Materials" Daneesh Simien

- "Facile Preparation of $\mathrm{Ag} / \mathrm{NiO}$ Composite Nanosheets and Their Antibacterial Activity" Cui-E Shi, Lu Pan, Yi He, Yong-Feng Wu, and Sai-Sai Xue

- "The Controlled Single-Step Synthesis of Ag/ $\mathrm{TiO}_{2}$ and $\mathrm{Au} / \mathrm{TiO}_{2}$ by Ultrasonic Spray Pyrolysis (USP)" J. Bogovic, R. Rudolf, and B. Friedrich

- "Tuning the Magnetic Properties of CobaltBased Metallic Glass Nanocomposites" Medha Veligatla, Santanu Das, Won Ki Lee, Junyeon Hwang, Orathai Thumthan, Yaowu Hao, and Sundeep Mukherjee

- "Influence of Pr Doping on the Structural, Morphological, Optical, Luminescent, and Nonlinear Optical Properties of RF Sputtered ZnO Films" R.Sreeja Sreedharan, R.Vinodkumar, I. Navas, Radhakrishna Prabhu, and V.P.Mahadevan Pillai

- "Synthesis and Characterization of High-Energy Ball Milled Nanostructured $\mathrm{Fe}_{25} \mathrm{Se}_{75}$ " A. Chebli, A. Djekoun, N. Boudinar, M. Benabdeslem, B. Bouzabata, A. Otmani, and J.J. Suñol

- "Thermal and Morphological Study of Epoxy Matrix with Chemical and Physical hybrid of Nanoclay/CarbonNnanotube" Elnaz Esmizadeh, Ali Akbar Yousefi, Ghasem Naderi, and Candida Milone

- "Synthesis of Graphene from Used Battery Electrode" Punith Kumar M K and Chandan Srivastava

- "Synthesis and Characterization of Covalently Linked Graphene/Chitosan Composites" S. Sayyar, E. Murray, S. Gambhir, G. Spinks, G.G. Wallace, and D.L. Officer 